\title{
On Piecewise Testable, Starfree, and Recognizable Picture Languages
}

\author{
Oliver Matz \\ Institut für Informatik und Praktische Mathematik \\ Christian-Albrechts-Universität Kiel, 24098 Kiel, Germany \\ e-mail: oma@informatik.uni-kiel.de
}

\begin{abstract}
We isolate a technique for showing that a picture language (i.e. a "two-dimensional language") is not recognizable. Then we prove the non-recognizability of a picture language that is both starfree (i.e., definable by means of union, concatenation, and complement) and piecewise testable (i.e., definable by means of allowed subpictures), solving an open question in [GR96].

We also define local, locally testable, and locally threshold testable picture languages and summarize known inclusion results for these classes. The classes of piecewise testable, locally testable, and locally threshold testable picture languages can, as in the word case, be characterized by certain (fragments of) first-order logics.
\end{abstract}

\section{Introduction}

In [GRST96,GR96], the authors investigated the class of recognizable picture language (as a straightforward generalization of recognizable word languages to two dimensions), and compared it to variants of classes of regular picture languages, defined by "regular expressions" built up by union, row- and columnconcatenation, and, optionally, iterated row-/column- concatenation and/or complement.

It turns out that the class of recognizable picture languages is not closed under complement, and the regular expressions without complement do not capture the class of recognizable picture languages, in contrast to the Kleene Theorem for the one-dimensional case. One question that remained open was whether every language defined by regular expressions with all of the above-mentioned operations is recognizable. We answer this question negatively, even for the case that the iterated concatenations are omitted, i.e. the "starfree" expressions. For this aim, we recapitulate and isolate a technique for showing the non-recognizability of a picture language. This technique has also been used in [MT97].

Besides, we consider some other adaptions of classes of formal languages to the two-dimensional case, namely different versions of first-order definable languages, as well as piecewise testable, locally testable, and locally threshold testable picture languages, and report some known and some simple results about these. For example, it is shown in [Wil97] that there is a first-order definable picture language that is not starfree. 


\section{Recognizable Picture Languages}

Throughout the paper, we consider a fixed alphabet $\Gamma$. A picture over $\Gamma$ is a matrix over $\Gamma$. By picture languages we refer to sets of pictures. The language of all pictures over $\Gamma$ is denoted by $\Gamma^{+,+}$. The language of all pictures of size $m \times n$ is denoted by $\Gamma^{m, n}$. There are two different, partial concatenations for pictures: the row concatenation $P$ (column concatenation $P Q$, respectively) of two pictures $P$ and $Q$ of the same width (height, respectively) is the picture obtained by appending $Q$ to the bottom (right, respectively) of $P$. These concatenations can be generalized to languages the straightforward way.

Since picture languages are the two-dimensional analogue to word languages, it is somewhat natural to try to transfer definitions of interesting word language classes to these. We will first give a straightforward definition of recognizability.

Definition 1. A picture language $L$ over $\Gamma$ is domino-local iff there are local word languages $L_{1}, L_{2}$ over $\Gamma$ such that $L$ is the set of pictures whose columns (considered as words) are in $L_{1}$ and whose rows are in $L_{2}$.

A picture language is recognizable if it is the image of a local picture language under some alphabet projection.

This definition is consistent with other equivalent definitions of recognizability given in [GRST96,GR96]. (Among these, there is the characterization via existential monadic second-order logic over the signature with the two binary relation symbols $S_{1}$ and $S_{2}$ for vertical and horizontal successors.)

The following fact has recently been proved by Klaus Reinhard.

Example 1. The set of all pictures over $\{a, b\}$ in which the set of $b$-positions is connected (where two $b$-positions are meant to be adjacent iff there are horizontally or vertically next to each other) is recognizable.

The complement of the above language is also recognizable, which is much easier to show.

Definition 2. For a picture language $L \subseteq \Gamma^{+,+}$and an integer $m \geq 1$, the fixed-height- $m$ word language of $L$, denoted by $L(m)$, is the following word language over $\Gamma^{m, 1}$ :

$$
L(m)=\left\{\left(\begin{array}{c}
a_{11} \\
\vdots \\
a_{m 1}
\end{array}\right) \cdots\left(\begin{array}{c}
a_{1 n} \\
\vdots \\
a_{m n}
\end{array}\right) \mid \begin{array}{ccc}
a_{11} \cdots & a_{1 n} \\
\vdots & & \vdots \\
a_{m 1} \cdots & a_{m n}
\end{array}\right\} L .
$$

The following lemma is formulated and proven in [MT97]. As far as the author knows, all arguments against recognizable languages depend on this lemma.

Lemma 1. Let $L \subseteq \Gamma^{+,+}$recognizable. Then there is a $k \geq 1$ such that for all $m \geq 1$ there is an $N F A \mathcal{A}$ with $k^{m}$ states that recognizes $L(m)$. 
Proof. Assume $L_{1}, L_{2}$, and $\Gamma$ are as in Definition 1. Let $m \geq 1$. The states of the constructed NFA are those columns of height $m$ that are, considered as words, in $L_{1}$, plus an additional initial state. The transitions and final states are chosen in such a way that each string of corresponding components of a run is in $L_{2}$. The transition labels are the images of the target states under the alphabet projection.

The following simple fact has been stated for example in [Bir96,GS96].

Lemma 2. Let $n \geq 1, L \subseteq \Gamma^{*}$ be recognizable by an $N F A$ with $n$ states. Let $M \subseteq \Gamma^{*} \times \Gamma^{*}$ such that

$$
\begin{gathered}
\forall(u, v) \in M: u v \in L, \\
\forall(u, v),\left(u^{\prime}, v^{\prime}\right) \in M:\left\{u v^{\prime}, u^{\prime} v\right\} \not L L .
\end{gathered}
$$

Then $|M| \leq n$.

The preceding two lemmas give the following result.

Lemma 3. Let $L \subseteq \Gamma^{+,+}$be recognizable. Let $\left(M_{m}\right)$ be a sequence with $\forall m$ : $M_{m} \subseteq \Gamma^{m,+} \times \Gamma^{m,+}$ and

$$
\begin{gathered}
\forall(P, Q) \in M_{m}: P Q \in L, \\
\forall(P, Q),\left(P^{\prime}, Q^{\prime}\right) \in M_{m}:\left\{P Q^{\prime}, P^{\prime} Q\right\} \nsubseteq L .
\end{gathered}
$$

Then $\left|M_{m}\right|$ is $2^{\mathcal{O}(m)}$.

Intuitively, this lemma says that for a recognizable picture language, there is no more than exponentially much space to pass information from one side of the picture to the other.

We use the above lemma to reformulate the proof of non-recognizability of an example language from [GRST96].

Proposition 1. Let $L$ be the set of pictures over $\{a, b\}$ of the form PP where $P$ is a square. Then $L$ is not recognizable.

Proof. For every $m \geq 1$ let $M_{m}:=\left\{(P, P) \mid P \in \Gamma^{m, m}\right\}$. We have for all squares $P, P^{\prime}$ that $P P^{\prime} \in L \Longleftrightarrow P=P^{\prime}$, so $\left(M_{m}\right)$ has the property of Lemma 3. But $\left|M_{m}\right|=2^{m^{2}}$ is not $2^{\mathcal{O}(m)}$, therefore $L$ is not recognizable.

In [GRST96] the non-recognizability of the above language has been shown using essentially the same argument. The complement of $L$ is recognizable, so a corollary is that the class of recognizable picture languages is not closed under complement.

In fact, the author does not know any example for a picture language whose non-recognizability can be shown, but not by this lemma.

We consider another example. 
Proposition 2. Let CORNERS be the set of pictures $P$ over $\{a, b\}$ such that whenever $P(i, j)=P\left(i^{\prime}, j\right)=P\left(i, j^{\prime}\right)=b$ then also $P\left(i^{\prime}, j^{\prime}\right)=b$. (Intuitively: Whenever three corners of a rectangle carry $a b$, then also the fourth one does.) CORNERS is not recognizable.

Proof. Let $n \geq 1$. For every partition $\mathcal{P}$ of $\{1, \ldots, 2 n\}$ into two-element sets we fix a bijection $\alpha_{\mathcal{P}}: \mathcal{P} \rightarrow\{1, \ldots, n\}$. (For example, we can choose $\alpha_{\mathcal{P}}\left(\left\{i, i^{\prime}\right\}\right)$ to be the number of elements $\left\{j, j^{\prime}\right\}$ of $\mathcal{P}$ for which $\min \left\{j, j^{\prime}\right\} \leq \min \left\{i, i^{\prime}\right\}$.)

Now we choose a picture $P$ over $\{a, b\}$ of size $2 n \times n$ such that for all $(i, j) \in$ $\{1, \ldots, 2 n\} \times\{1, \ldots, n\}$ :

$$
P(i, j)=b \Longleftrightarrow \exists i^{\prime}:\left\{i, i^{\prime}\right\} \in \mathcal{P} \wedge j=\alpha_{\mathcal{P}}\left(\left\{i, i^{\prime}\right\}\right) .
$$

Let $M_{n}$ be the set of all pairs $\left(P_{\mathcal{P}}, P_{\mathcal{P}}\right)$ where $\mathcal{P}$ is a partition of $\{1, \ldots, 2 n\}$ into two-element sets.

Then we have for all partitions $\mathcal{P}, \mathcal{P}^{\prime}$ that $P_{\mathcal{P}} P_{\mathcal{P}^{\prime}} \in$ CORNERS $\Longleftrightarrow \mathcal{P}=$ $\mathcal{P}^{\prime}$, so $\left(M_{n}\right)$ has the property of Lemma 3 .

For the number $A_{n}$ of partitions of $\{1, \ldots, 2 n\}$ into two-element sets one easily verifies the recursion formula $A_{1}=1, A_{n+1}=(2 n+1) A_{n}$. We have that $\left|M_{n}\right|=A_{n} \geq n$ ! is not $2^{\mathcal{O}(n)}$ and hence Lemma 3 implies that CORNERS is not recognizable.

\section{Piecewise Testable Picture Languages}

Definition 3. Let $P \in \Gamma^{m, n}$ and $Q \in \Gamma^{+,+}$. Then $P$ is a subpicture of $Q$ if there are strictly monotone functions $f:\{1, \ldots, m\} \rightarrow \mathbb{N}_{\geq 1}$ and $g:\{1, \ldots, n\} \rightarrow$ $\mathbb{N}_{\geq 1}$ such that $Q(f(i), g(j))=P(i, j)$ for all $(i, j) \in\{1, \ldots, m\} \times\{1, \ldots, n\}$.

Let $m, n \in \mathbb{N}_{\geq 1}$. Two pictures $Q_{1}, Q_{2}$ are $(m, n)$-equivalent $\left(Q_{1} \sim_{m n} Q_{2}\right.$ for short) iff they have the same subpictures of size $m \times n$. A picture language $L$ is piecewise testable iff there is some $(m, n)$ such that $L$ is a union of $\sim_{m n^{-}}$ equivalence classes.

Example 2. The picture language CORNERS from Proposition 2 is piecewise testable.

The proof is immediate since CORNERS is the set of pictures such that no $2 \times 2$ subpicture of $P$ has exactly $3 b$ 's, and this property holds for every or for none element of a $(2,2)$-equivalence class.

This example shows that, unlike in the theory of formal word languages, not every piecewise testable picture language is recognizable.

Remark 1. The class of piecewise testable picture languages is characterized by Boolean combinations of existential first-order formulas with the two binary predicates $\leq_{1}, \leq_{2}$.

The proof is similar to the word case. 
Example 3. Let CROSS be the language of all pictures over $\{a, b\}$ containing

$$
\begin{aligned}
& a b a \\
& b \quad b \quad b \\
& a b a
\end{aligned}
$$

as a subpicture. CROSS is piecewise testable.

\section{Starfree Picture Languages}

Definition 4. The class of starfree picture languages over $\Gamma$ is given by the smallest set that contains all finite picture languages over $\Gamma$ and is closed under row- and column concatenation, finite union, and complement.

The class of recognizable picture languages is closed under row- and column concatenation and union, but (as mentioned before) not under complement. In [GRST96] the authors asked whether, nevertheless, every starfree picture language is recognizable. We answer this question negatively.

Proposition 3. The picture language CORNERS from Proposition 2 is starfree.

Proof. Let $K:=\bigcup\left(\begin{array}{c}(w(\sim \emptyset) x) \\ (\sim \emptyset) \\ (y(\sim \emptyset) z)\end{array}\right)$, where the union ranges over all quadruples $(w, x, y, z) \in\{a, b\}^{4}$ such that $w x y z \in b^{*} a b^{*}$, and $\sim$ denotes complement w.r.t. $\{a, b\}^{+,+}$. Then $K$ is the set of all pictures over $\{a, b\}$ such that exactly one of the corners carries an $a$.

Clearly, $\left(\begin{array}{c}((\sim \emptyset) K(\sim \emptyset)) \\ (\sim \emptyset)\end{array}\right)$ is the complement of $L$, so $L$ is starfree.

The following is shown in [Wil97]:

Lemma 4. The language CROSS from Example 3 is not starfree.

\section{Local, Locally Testable, and Locally Threshold Testable Picture Languages}

We give straightforward adaptions of definitions of languages classes defined by certain "local" properties. These definitions can also be found, for instance, in [GRST96].

Definition 5. Let $P \in \Gamma^{m, n}$ and $Q \in \Gamma^{m^{\prime}, n^{\prime}}$. Then $P$ is a subblock of $Q$ if there are $k \leq m^{\prime}-m$ and $l \leq n^{\prime}-n$ such that $Q(i, j)=P(k+i, l+j)$ for all $(i, j) \in\{1, \ldots, m\} \times\{1, \ldots, n\}$.

For a picture $P$ over $\Gamma$, we denote by $\hat{P}$ the picture over $\Gamma \cup\{\#\}$ that results from $P$ by surrounding it with the fresh boundary symbol \#. 
A picture language $L$ is local iff there is some set $\Delta$ of $2 \times 2$-pictures over $\Gamma \cup\{\#\}$ such that $L$ contains exactly those pictures $P$ for which the $(2 \times 2)$ subblocks of $\hat{P}$ are in $\Delta$.

Let $m, n \in \mathbb{N}_{\geq 1}$. Two pictures $Q_{1}, Q_{2}$ are $(m, n)$-block-equivalent $\left(Q_{1} \cong_{m n}\right.$ $Q_{2}$ for short) iff $\hat{Q}_{1}$ and $\hat{Q_{2}}$ have the same set of subblocks of size $m \times n$. A picture language $L$ is locally testable iff there is some $(m, n)$ such that $L$ is a union of $(m, n)$-block-equivalence classes.

Let $d, t \geq 1$. Two pictures $Q_{1}, Q_{2}$ are $(d, t)$-block-threshold-equivalent iff for every square picture $P$ of size $d^{\prime} \times d^{\prime}$ (with $d^{\prime} \leq d$ ), the numbers of occurrences of $P$ as a subblock in $\hat{Q}_{1}$ (respectively $\hat{Q}_{2}$ ) are equal or both $>t$. A picture language is locally threshold testable iff there are $d, t$ such that $L$ is a union of $(d, t)$-block-threshold-equivalence classes.

Since every local language is a union of $(2,2)$-block-equivalence classes, and $(m, n)$-block-equivalence is coarser than $(\max \{m, n\}, 0)$-block-threshold-equivalence, we have that every domino-local language is local, every local language is locally testable, and every locally testable picture language is locally threshold testable.

In [GR96] it is shown that the class of recognizable picture languages is the class of those picture languages that can be obtained from a local picture language via alphabet projection.

Remark 2. 1. The class of locally threshold testable picture languages is characterized by first-order logic over the signature $\left\{S_{1}, S_{2}\right\}$ with two binary relation symbols $S_{1}, S_{2}$ for the two successor relations.

2. The class of locally testable picture languages is characterized by Boolean combinations of existential first-order sentences over the signature $\left\{S_{1}, S_{2}\right.$, left, right, top, bottom , where the latter four predicates are unary and say that a position is at the respective border.

The first statement is shown in [GRST96] and the second can be proved similarly to the case of word languages.

\section{An Overview of Language Classes and Open Questions}

Let us denote the classes of recognizable, piecewise testable, starfree, local, and first-order definable ${ }^{1}$ picture languages by $R E C, P T, S F, L O C$, and $F O\left(\leq_{1}, \leq_{2}\right)$, respectively. We have the inclusion diagram presented in the following figure, where lines indicate proper inclusions and non-connected classes are incomparable for non-trivial alphabets.

${ }^{1}$ in the sense of [Wil97], i.e., over the signature with binary relation symbols $\leq_{1}$ and $\leq_{2}$ for vertical and horizontal orderings 


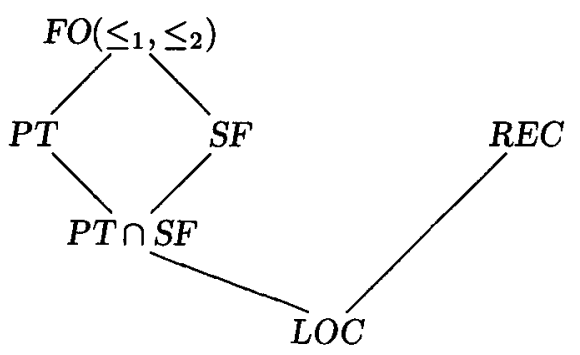

(Simple proofs show that every starfree and every piecewise testable picture language is first-order definable. This infers e.g. $F O\left(\leq_{1}, \leq_{2}\right) \nsubseteq \mathbb{R E C}$, correcting a mistake in [Mat95]. The non-inclusion results $R E C \nsubseteq F O\left(\leq_{1}, \leq_{2}\right)$ and $F O\left(\leq_{1}, \leq_{2}\right) \& P T$, and $S F \nsubseteq P T$ carry over from the theory of formal word languages because when restricted to pictures of height one, each of these classes equals the respective class of word languages.)

If we denote the classes of locally testable and locally threshold testable picture languages by $L T$ and $L T T$, respectively, we have the following inclusion chain

$$
L O C \subsetneq L T \subsetneq L T T \subsetneq F O\left(\leq_{1}, \leq_{2}\right) .
$$

Here, the non-inclusions are again witnessed by the well-known examples from word language theory, and the last inclusion is trivially inferred by the logical characterizations of Remark 2.

Since $R E C$ is not closed under complement (as mentioned after Proposition 1) whereas the classes $F O\left(\leq_{1}, \leq_{2}\right), P T$, and $S F$ are, the class co-REC of complements of recognizable picture languages is incomparable to all of these classes, too.

Concluding, one could say that in the world of picture languages, only trivial language class inclusions hold. (Unlike in the theory of word languages, where we have $P T \subsetneq S F=F O \subsetneq R E C$.) Another justification for this statement is the fact that also the class of context-free picture languages (as defined in [Mat97]) is incomparable to every other class of picture languages mentioned here (including $L O C)$.

One open question is: Is there a natural example for a non-recognizable picture language for which Lemma 3 fails to prove the non-recognizability? One candidate is the language of squares over $\{a, b\}$ that have as many $a$ 's as $b$ 's. It is easy to see that Lemma 3 cannot be used to show the non-recognizability of this example language, however we conjecture that it is not recognizable.

\section{References}

[Bir96] Jean-Carnille Birget. The state complexity of $\overline{\Sigma^{*} \bar{L}}$. Information Processing Letters, 58:185-188, 1996.

[GR96] D. Giammarresi and A. Restivo. Two-dimensional languages. In G. Rozenberg and A. Salomaa, editors, Handbook of Formal Language Theory, volume III. Springer-Verlag, New York, 1996. 
[GRST96] D. Giammarresi, A. Restivo, S. Seibert, and W. Thomas. Monadic secondorder logic and recognizability by tiling systems. Information and Computation, 125:32-45, 1996.

[GS96] Ian Glaister and Jeffrey Shallit. A lower bound technique for the size of nondeterministic finite automata. Information Processing Letters, 125:3245, 1996.

[Mat95] Oliver Matz. Klassifizierung von Bildsprachen mit rationalen Ausdrücken, Grammatiken und Logik-Formeln. Diploma thesis, Christian-Albrechts-Universität Kiel, 1995. (German).

[Mat97] Oliver Matz. Regular expressions and context-free grammars for picture languages. In Rüdiger Reischuk, editor, STACS'97, volume 1200 of Lect. Notes Comput. Sci., pages 283-294, Lübeck, Germany, 1997. Springer-Verlag.

[MT97] Oliver Matz and Wolfgang Thomas. The monadic quantifier alternation hierarchy over graphs is infinite. In Twelfth Annual IEEE Symposium on Logic in Computer Science, pages 236-244, Warsaw, Poland, 1997. IEEE.

[Wil97] Thomas Wilke. Star-free picture expressions are strictly weaker than firstorder logic. In Pierpaolo Degano, Roberto Gorrieri, and Alberto MarchettiSpaccamela, editors, Automata, Languages and Programming, volume 1256 of Lect. Notes Comput. Sci., pages 347-357, Bologna, Italy, 1997. Springer. 